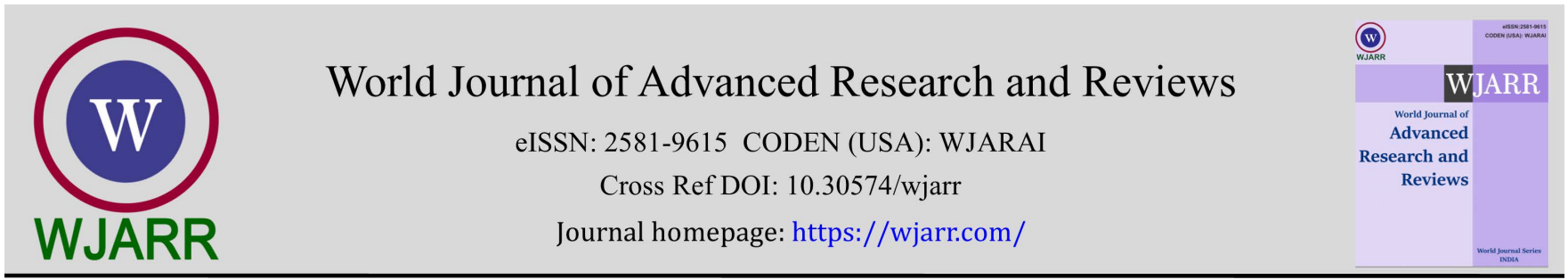

(CASE REPORT)

Check for updates

\title{
Management of an Alveolar paratesticular Rhabdomyosarcoma: A case report
}

\author{
Zenab Alami 1,2, , Amina Bouziane 1,2, Wissal Hassani 1,2, Fatima Zahrae Farhane ${ }^{1,2}$ and Touria Bouhafa 1,2 \\ ${ }^{1}$ Faculty of Medecine and pharmacy of FES, Sidi Mohammed Ben-Abdellah University, MOROCCO. \\ ${ }^{2}$ Radiatherapy Department, Oncology hospital, Hassan II University Hospital, FES, MOROCCO.
}

World Journal of Advanced Research and Reviews, 2021, 12(02), 598-602

Publication history: Received on 10 October 2021; revised on 21 November 2021; accepted on 23 November 2021

Article DOI: https://doi.org/10.30574/wjarr.2021.12.2.0602

\begin{abstract}
Paratesticular Rhabdomyosarcoma is a rare mesenchymal tumor. The Alveolar variant is the one with the worse prognosis. It comprehend $20 \%$ of cases.

The treatment is multimodal combining surgery, chemotherapy and radiotherapy. Depending on the extent of disease and the staging group, the approach of treatment is different.

Here we report the case of an alveolar Paratesticular rhabdomyosarcoma and review the literature regarding this unusual clinical entity.
\end{abstract}

Keywords: Rhabdomyosarcome; Paratesticular; Staging; Surgery; Radiotherapy

\section{Introduction}

Rhabdomyosarcoma is a rare mesenchymal tumor. Paratesticular location is the most common urogenital location. It is a tumor that mainly interests children and remains exceptional in adults. Several forms are described and the Alveolar variant is the one with the worse prognosis. It comprehend $20 \%$ of cases. The treatment is multimodal combining surgery, chemotherapy and radiotherapy.

We report the case of a 9-years-old boy admitted for appearance of a tumor in the right scrotum.

\section{Case presentation}

E.A 9 years old, 3rd of a siblings of 4 children, resulting from a 1st degree consanguineous marriage of a mother aged 39 and a father aged 43, the child had no particular pathological history, consults for a large purse associated with moderate pain evoluating for a year and gradually increasing in volume. The clinical examination found a patient in a good general condition, apyretic, a right inguinoscrotal swelling of hard consistency, painless, mobile, opaque and associated with a hydrocele.

The ultrasound performed on admission shows a heterogeneous right intra-scrotal tissue mass, of irregular contours, taking the doppler color, measuring $2.7 * 1.7 \mathrm{~cm}$ associated with a hydrocele of great abundance with echogenic content, the right and left testis are intra-scrotal, oval in shape of homogeneous echostructure.

Thoraco-abdomino-pelvic Computed Tomography (CT-TAP) as part of the extension assessment, showed a right intrascrotal testicular tissue process, roughly oval with irregular contours, hypodense in contrast, spontaneously enhanced

\footnotetext{
* Corresponding author: Zenab Alami

Faculty of Medecine and pharmacy of FES, Sidi Mohammed Ben-Abdellah University, MOROCCO.
}

Copyright $@ 2021$ Author(s) retain the copyright of this article. This article is published under the terms of the Creative Commons Attribution Liscense 4.0 
significantly after injection of contrast, and measuring $29 * 26 * 27 \mathrm{~mm}$. This mass comes into intimate contact with the contralateral testis. At the bottom it respects the penis before it reaches the skin, it's associated with an abundant homolateral hydrocele and a nodular scrotal thickening (figure 1). Absence of lymph node or distant metastases. The tumor markers were normal (alpha FP, BHCG, LDH).
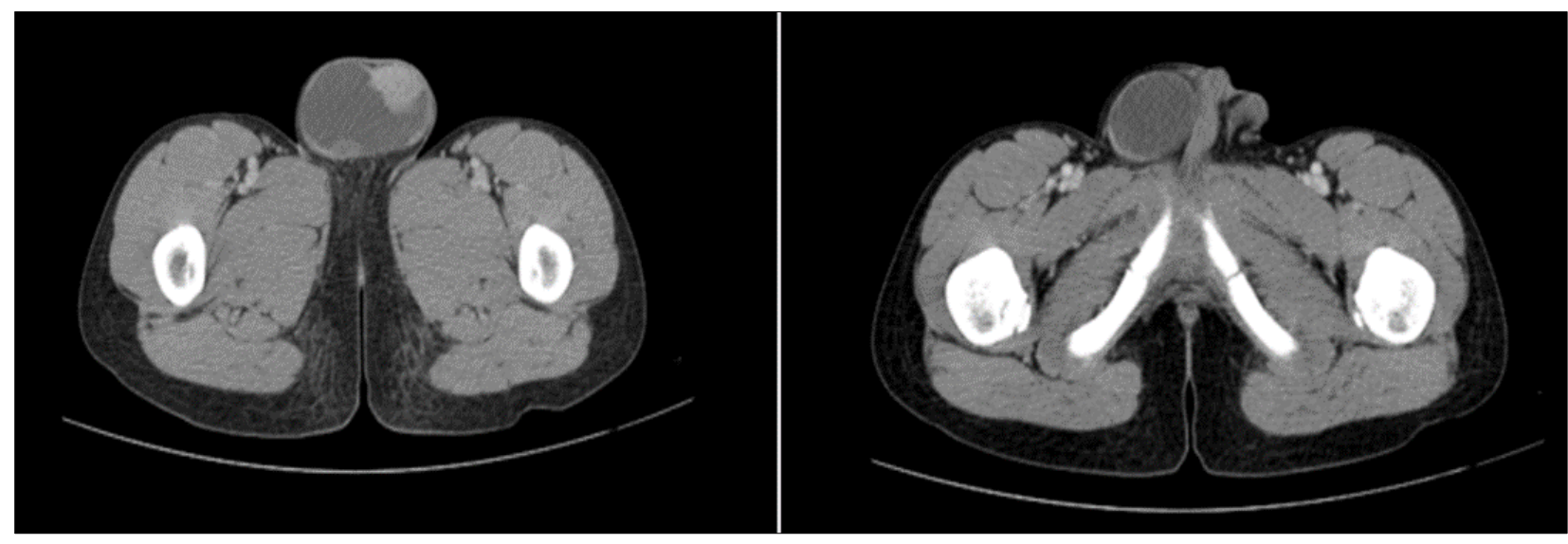

Figure 1 Pre-operative Thoraco-abdomino-pelvic CT showing a right intra-scrotal testicular tissue process, measuring $29 * 26 * 27$, this mass comes into intimate contact with the contralateral testis. At the bottom it respects the penis before it reaches the skin, it's associated with an abundant homolateral hydrocele.

Radical orchiectomy through an inguinal incision with high dissection and ligation of the spermatic cord was performed. No retroperitoneal lymph node dissection (RPLND) have been performed.

The mass measured $9.5 * 5.5 * 4.5 \mathrm{~cm}$. On macroscopic examination, a homogeneous whitish tumor of firm consistency. Microscopic examination and proliferation of tumors arranged in a sheet, massive and spans. The tumor cells are rounded, sometimes oval in appearance, with enlarged nuclei and abundant and eosinophilic cytoplasm. Mitosis is frequent. The stroma is fibrous myxoid in focus, the limit of the spermatic cord is healthy, absence of vascular emboli.

An immunohistochemical supplement was carried out to support the diagnosis, the tumor cells express desmine in a diffuse manner and myogenin by more than 50\%, and they do not express CD117 which eliminates a seminoma, CD30 which eliminates embryonic carcinoma and anaplastic lymphoma, CD45 which eliminates lymphoma, CK and EMA which eliminates carcinoma or inhibin. Immunostaining has revealed alveolar rhabdomyosarcoma.

After a multidisciplinary consultation meeting, the disease was classified as high risk (group G) according to "EpSSG non metastatic RMS study 2005."

The patient received chemotherapy according to IVA regimen (Ifosfamide $3000 \mathrm{mg} / \mathrm{m}^{2} /$ day, Vincristine $1.5 \mathrm{mg} / \mathrm{m} 2$, Actinomycin D $1.5 \mathrm{mg} / \mathrm{m} 2 /$ day). An evaluation was done after the 4th course based on a thoraco-abdomino-pelvic CT scan, which did not show tumor infiltration or distant metastasis. No further surgery has been accomplished, and a total of 14 cycles were delivered.

External beam RT to hemiscrotum was started after the 4th course of chemotherapy and after a Contralateral testicle transposition with a total dose of 41, 4Gy.

After a follow up of 18 months, the children have no evidence of disease.

\section{Discussion}

Paratesticular rhabdomyosarcoma (PT-RMS) accounts for $7 \%$ to $10 \%$ of all genitourinary tract RMS tumors and is the third most common, following RMS of the prostate and bladder. There are two frequency peaks found for the development of rhabdomyosarcoma the first at 1 to 5 years and then at 16 years of age [1].

The consensus is that this tumor derives from mesenchymal elements of the testis envelope, epididymis and spermatic cord. Its clinical discovery is often fortuitous by a painless intrascrotal mass. 
The testicular examination should be bilateral and careful. It eliminates the main causes of painless large bursa including hydrocele, cord cyst, epididymitis or inguino-scrotal hernia which could lead to detrimental delay in diagnosis [2, 3]. The paratesticular site of the tumor is difficult to determine by palpation of the scrotum [4], the test is being difficult to identify. A clinical examination should be complemented by an examination of the lymph nodes and a general examination to investigate metastases.

Scrotal ultrasonography is the initial imaging modality of choice. The sonographic description of PT-RMS varies in the literature but common findings include a highly reflective mass with increased vascularity on color Doppler [5]. The dosage of testicular tumor markers including alpha-fetoprotein, beta-human chorionic gonadotropin and carcinoembryonic antigen is systematically indicated in the presence of a testicular mass. In rhabdomyosarcoma, they are usually normal [6].

Once the diagnosis of PT-RMS is established, proper clinical staging is required. The most common staging modality is cross-sectional imaging with computed tomography (CT). A thoraco-abdomino-pelvic CT scan is performed to assess for retroperitoneal and mediastinal lymphadenopathy along with distant metastases. The risk of bone and bone marrow metastases increases with clinical stage and is dependent on tumor histology [7].

RMS has been traditionally classified into 3 main histologic subtypes: embryonal (ERMS), alveolar (ARMS), and pleomorphic. Additional variants have been recently identified, such as spindle cell RMS and sclerosing RMS comprising only $5 \%$ to $10 \%$ of RMS $[8,9]$.

The ERMS and ARMS are the most prevalent and comprehend $70 \%$ and $20 \%$ of cases, respectively. Of these, ARMS is the one with the worse prognosis $[10,11]$. However, the majority of authors agree on the fact that the early diagnosis and surgical treatment determine the prognosis [12].

RMS staging is multifactorial and outcomes are reported based on 3 different classifications [6]:

- Stage: Determined from pretreatment data based on location of primary site, tumor size (widest diameter), presence or absence of regional lymph node, or distant metastases.

- Group: Determined by local tumor status based on postsurgical resection or biopsy, with pathologic assessment of the tumor margin and of lymph node disease.

- $\quad$ Risk category: A combination of stage, group, and tumor histology.

The therapeutic strategy depends on the stage of the tumor and the prognostic group. It must be decided in a multidisciplinary consultation meeting. All patients require multimodality therapy consisting of surgery (if possible) followed by chemotherapy \pm Radiotherapy (RT).

Our patient had a Non metastatic alveolar RMS of a favorable site, Stage 1 According to IRS preoperative staging system, groupe IIA Accoridng to IRS surgical-pathologic grouping system. all these criteria classified our patient as an intermediat risk group. Therefore, he had a radical orchiectomy through an inguinal incision with high dissection and ligation of the spermatic cord as recommended. We did not perform retroperitoneal lymph node dissection (RPLND) because of the absence of radiological lymph node involvement. Since the publication of the International Society of Pediatric Oncology (SIOP), this systematic paraortic lymph node dissection has been controversial and its interest in localized forms has been discussed due to the efficacy of chemotherapy on lymph node micrometastases [13]. In addition, the significant morbidity of this dissection must be weighed against the risk of recurrence, therefore it is currently recommended not to perform lymphadenectomy in the absence of lymph node involvement demonstrated by the CT scan.

Chemotherapy should be routinely administered since rhabdomyosarcoma is chemosensitive. Chemotherapy agents include Vincristine, actinomycin D, cycophosphamide, topotecan, and irinotecan. The most widely used chemotherapy protocol is based on the combination Vincristine-actinomycin D-cycophosphamide. For low, intermediate, and high-risk patients the duration is 16,36 , and 46 weeks according to SIOP protocols [14].

Radiation therapy (RT) has assumed a major role in the management of RMS. The use of RT has been limited to treatment of the ipsilateral scrotum, most commonly as salvage treatment of nodal extension. It is also used in cases of prior trans-scrotal resection or biopsy and in the rare event of positive margins after surgical excision.

RT dose depends on stage and group, but, in general, 0-36 Gy for complete resection, 36 Gy for microscopic residual and N0, 41.4 Gy for microscopic residual and N1, and 50.4 Gy for gross residual. 
Alveolar PT-RMS is uncommon but has shown to have an improvement in Failure Free Survival with RT. Wolden et al. [15] found that patients with alveolar histology had a significant improvement in 10-year FFS rate when treated with RT compared to chemotherapy alone (77\% vs. 44\%, P 1/4 0.02).

If scrotal RT, including intensity-modulated RT (IMRT), is required, then temporary transposition of the remaining normal testis should be done to allow a good endocrine testicular function preservation [16].

in our case, Contralateral testicle was transposed near the ipsilateral anterior superior iliac spine prior to RT and later reimplanted.

The prognosis of paratesticular rhabdomyosarcoma depends on tumor stage, histological type and response to treatment [17]. Currently, the overall survival rate of these tumors has impressively increased. The 5-year survival rate varies from 80 to $88.5 \%$ in the localized forms, to $22.2 \%$ in the metastatic forms $[18,19]$.

\section{Conclusion}

Paratesticular rhabdomyosarcoma is a rare pathology, but with extremely serious metastatic potential. The main challenge is to diagnose this disease early enough to promote curative treatment.

\section{Compliance with ethical standards}

\section{Acknowledgments}

This research did not receive any specific grant from funding agencies in the public, commercial, or not-for-profit sectors.

\section{Disclosure of conflict of interest}

The authors have no conflicts of interest to disclose.

\section{Statement of ethical approval}

The present research work does not contain any studies performed on animals/humans subjects by any of the authors.

\section{Statement of informed consent}

Informed consent was obtained from all individual participants included in the study.

\section{References}

[1] Ahmed HU, Arya M, Muneer A, et al. Testicular and paratesticular tumors in the prepubertal population. Lancet Oncol. 2010; 11: 476-83.

[2] Masson BJ, Kier R: Sonographic and MR imaging appearances of paratesticular rhabdomyosarcoma. AJR Am J Roentgenol. 1998; 171: 523-524.

[3] Trippitelli A, Rosi P, Selli C, Carini M, Turini D: Rhabdomyosarcoma of spermatic cord in adult. Urology. 1982; 19: 533-535.

[4] Slama A, Jaidane M, Ben Sorba N, et al. Le rhabdomyosarcome paratesticulaire. Prog Urol. 2000; 10: 1232-4.

[5] Mak CW, Chou CK, Su CC, et al. Ultrasound diagnosis of paratesticular rhabdomyosarcoma. Br J Radiol. 2004; 77: 250-2.

[6] Pankaj P Dangle, MD MCh, Andres Correa MD, Lauren Tennyson MD, Bishoy Gayed MD, Miguel Reyes-Múgica, MD, Michael Ost MD. Current management of paratesticular rhabdomyosarcoma Urologic Oncology: Seminars and Original Investigations. 2015; $1-9$.

[7] Weiss AR, Lyden ER, Anderson JR, et al. Histologic and clinical characteristics can guide staging evaluations for children and adolescents with rhabdomyosarcoma: a report from the Children's Oncology Group Soft Tissue Sarcoma Committee. J Clin Oncol. 2013; 31: 3226-32. 
[8] Fletcher CDM, Bridge JA, Hogendoorn PCW, et al. WHO classi- fication of tumours of soft tissue and bone. Lyon, France: IARC Press. 2013; 134-5.

[9] Ferrari A, Bisogno M, Casanova C, et al. Paratesticular Rhabdomyosarcoma: report from the Italian and German Cooperative Group. J Clin Oncol. 2002; 20: 449-55.

[10] M Ciesla, J Dulak, A Józkowicz. MicroRNAs and epigenetic mechanisms of rhabdomyosarcoma development. Int J Biochem Cell Biol. 2014; 53: 482-492.

[11] DM. Parham, F.G. Barr. Classification of rhabdomyosarcoma and its molecular basis. Adv Anat Pathol. 2013; 2 : 387-397

[12] Faure A, Diakité ML, Panait N, Chaumoître K, Rome A, Merrot T. Le rhabdomyosarcome para-testiculaire de l'enfant: une urgence scrotale. Arch Pediatr. Dec 2012; 19(12): 1340- 4

[13] Boris Amougou et al. Rhabdomyosarcome paratesticulaire: à propos d'un cas et revue de la littérature. Pan African Medical Journal. 2021; 39(71).

[14] Wu HY, Snyder HM, Womer RB. Genitourinary rhabdomyosarcoma: which treatment, how much, and when? J Pediatr Urol. 2009; 5: 501-6.

[15] Wolden SL, Anderson JR, Crist WM, et al. Indications for radio- therapy and chemotherapy after complete resection in rhabdomyosarcoma: A report from the Intergroup Rhabdomyosarcoma Studies I to III. J Clin Oncol. 1999; 17: 3468-75.

[16] Le Bon M, Lejeune $\mathrm{H}$, Helfre $\mathrm{S}$, et al. Testicular transposition in children before scrotal external radiotherapy. Pediatr Blood Cancer. 2020; 67(9): e28526.

[17] Meza JL, Anderson J, Pappo AS, et al. Analysis of prognostic factors in patients with nonmetastatic rhabdomyosarcoma treated on intergroup rhabdomyosarcoma studies III and IV: the children's oncology group. J Clin Oncol. 2006; 24: 3844-51.

[18] Stevens MC, Rey A, Bouvet N, et al. Treatment of nonmetastatic rhabdomyosarcoma in childhood and adolescence: third study of the international society of paediatric oncology-SIOP malignant mesenchymal tumor 89. J Clin Oncol. 2005; 23: 2618-28.

[19] Ferrari A, Casanova M, Massimino M, et al. The management of paratesticular rhabdomyosarcoma a single institutional experience with 44 consecutive children. J Urol. 1998; 159: 1031-4. 